\title{
Retour à Solutré \\ Route de la Roche : fouille préventive d'un gisement de plein air magdalénien
}

\author{
Jean-Baptiste Lajoux, Romain Malgarini, Céline Bemilli et Grégory Bayle
}

\author{
Avec la collaboration de P. Alix, P. Bertran, C. Bontemps, E. Claud, C. Fossurier, \\ M. Lagache, P. Listrat, C. Peschaux et F. Sellami.
}

\section{Un siècle et demi de recherche en Préhistoire}

Solutré (Saône-et-Loire, Bourgogne Franche-Comté, fig. 1.1), site emblématique de la Préhistoire européenne, fait l'objet depuis sa découverte en 1866 par A. Arcelin et H. Testot-Ferry, de fouilles successives sur le secteur du "Crot du Charnier» (Combier et Montet-White, 2002 ; Bemilli et Bayle, 2009; Bayle et al., 2011 ; Connet et al., 2012). En périphérie, des ramassages de surface et des suivis de travaux témoignent d'une occupation du versant sud de la Roche depuis le Paléolithique moyen (Combier et Montet-White, 2002 ; fig. 1.2). La fouille « Route de la Roche » offre l'opportunité unique de documenter une occupation du Magdalénien sur le versant, en marge du gisement classique.

\section{Du diagnostic à la fouille : un potentiel sous évalué}

La fouille préventive " Route de la Roche » fait suite à un diagnostic archéologique réalisé entre le 18 et 22 mai 2015, dans le cadre d'un projet de construction d'une maison individuelle. L'évaluation a été établie à partir des observations menées dans trois tranchées de sondages, implantées en fonction de la configuration du terrain et de ses contraintes. Les surfaces ouvertes représentaient une superficie totale de 41,6 $\mathrm{m}^{2}$, soit plus de $12 \%$ de la parcelle. Deux tranchées ont permis l'identification d'un niveau archéologique attribué au Magdalénien moyen, sur la base de l'industrie lithique et d'une date radiocarbone à $15230 \pm 50$ BP (Beta 412745; Lajoux, 2015). L'ensemble de la séquence stratigraphique a pu faire l'objet d'un premier examen qui écartait la présence de niveaux plus anciens. Le matériel archéologique, la faune en particulier, se caractérisait par une forte altération des états de surface (Bemilli, 2015). Quelques déchets d'industrie osseuse (bois de renne), également dégradés, ont pu être isolés (Malgarini, 2015). À l'issu du diagnostic, les données permettaient de conclure à la présence d'un niveau archéologique cohérent, de densité moyenne voire faible $\left(65\right.$ pièces $\left./ \mathrm{m}^{2}\right)$, présent sur la moitié sud de la parcelle, et justifiant une prescription d'une fouille (Lajoux, 2015).

Étant donné la richesse du gisement, la fouille initialement programmée du 19 octobre au 18 décembre 2015 s'est prolongée jusqu'au 25 avril 2016. Le site s'est révélé plus complexe que ce que les observations du dia- gnostic laissaient supposer. Le niveau archéologique observé dans les tranchées de sondage correspondait en réalité à des lambeaux remaniés en périphéries d'un niveau principal non atteint lors du diagnostic. La surface fouillée, qui atteint au total $70 \mathrm{~m}^{2}$, se répartit sur 2 secteurs : l'ensemble « Ouest», d'extension limitée à 15 $\mathrm{m}^{2}$ et l'ensemble «Est » où se développe le niveau principal et qui s'étend sur $55 \mathrm{~m}^{2}$ (fig. 1.3). La mise en place d'un abri couvrant l'intégralité de la surface de fouille, et les moyens matériels mis à disposition (alimentation électrique, éclairage, chauffage, ...) ont pleinement participé au bon déroulement de l'opération jusqu'à son terme.

\section{Les premières données de terrain}

Sont présentés ici les premières données brutes de terrain et quelques éléments qui témoignent de la qualité et de l'intérêt du gisement. Si la quantité de faune et de matériel lithique, ainsi que la présence de restes humains, contribuent au caractère exceptionnel du site, son originalité repose sur un ensemble d'industrie osseuse remarquable et l'abondance des éléments de parure. Les données recueillies sont également en mesure de définir précisément le cadre chronostratigraphique et les conditions taphonomiques spécifiques propres à ce gisement de plein air de versant.

\section{Stratigraphie et taphonomie}

La séquence stratigraphique, épaisse de $3 \mathrm{~m}$, se développe sur le substrat marneux jurassique (Lias/Dogger). Elle est formée d'une succession de dépôts alimentés par la dégradation de la corniche calcaire située plus haut, où s'intercalent des lentilles de marnes remaniées. La morphologie irrégulière du versant, est marquée de dépressions et de bourrelets résultant de mouvements dans les marnes.

Les couches archéologiques se divisent en deux ensembles distincts :

- l'ensemble « Ouest » constitué par une couche remaniée et résiduelle (C-19), à mettre en relation avec une structure de combustion située en amont (fig. 3);

- l'ensemble «Est» structuré en trois unités : couche $4 / 22$, couche 13 et couche 14 . Leurs épaisseurs varient entre 5 et $30 \mathrm{~cm}$. Les dynamiques de solifluxion semblent être à l'origine du dédoublement $(\mathrm{C}-4 / 22)$ et des déformations (C-13) du niveau principal (C-14). Si, d'une manière générale, la persistance des connexions 
1
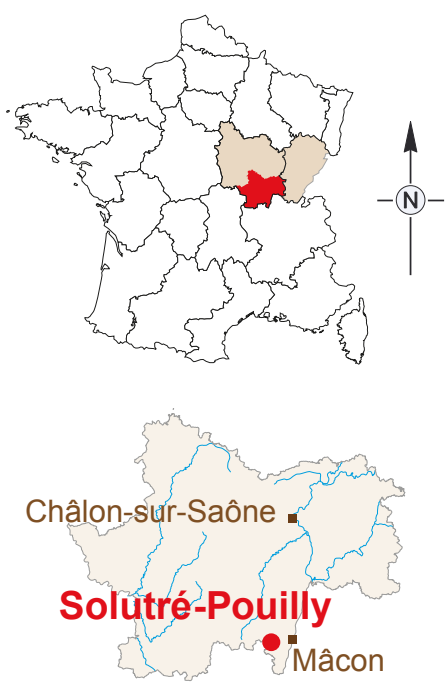

échelle $1 / 5500000$

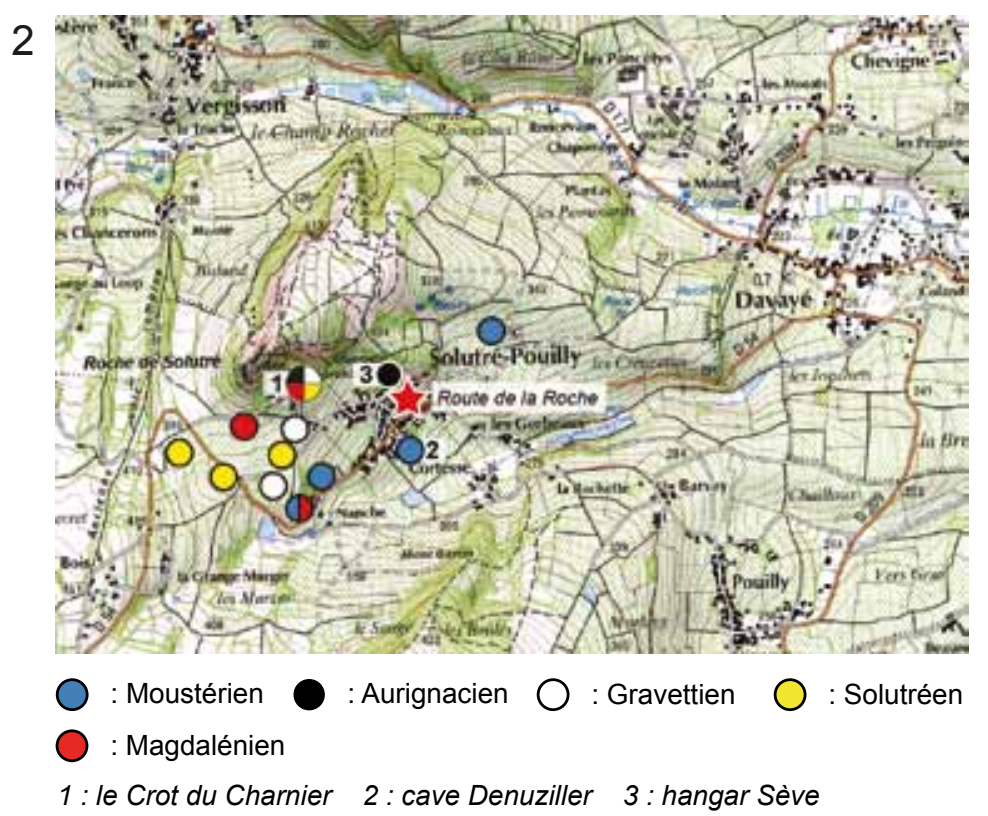

1 :le Crot du Charnier 2 : cave Denuziller $3:$ hangar Sève

3

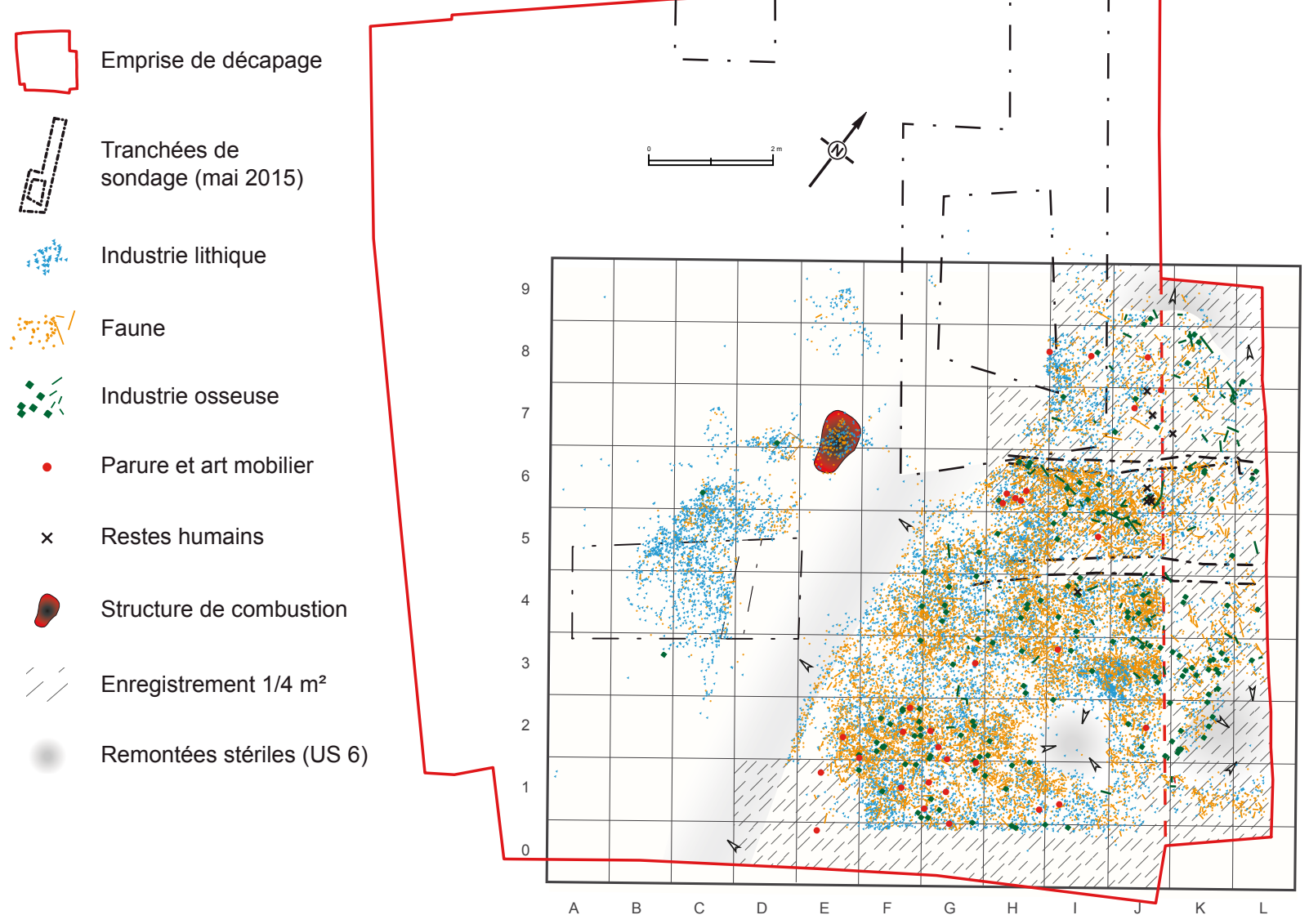

Fig. 1 - 1 : situation géographique de Solutré (D. Watts, INRAP); 2 : position du gisement « Route de la Roche » et des autres gisements préhistoriques de Solutré (fond de carte topographique, IGN); 3 : plan de répartition des vestiges du gisement "Route de la Roche » (relevés topographiques, équipe INRAP; DAO M. Lagache, J.-B. Lajoux, R. Malgarini). 
anatomiques et l'absence de tri granulométrique au sein du matériel illustrent l'action modérée des processus post-dépositionnels sur le niveau principal, l'orientation préférentielle des éléments allongés suggèrent une redistribution du matériel sur certains secteurs.

\section{Le matériel archéologique en quelques chiffres}

Compte tenu des conditions d'intervention, l'enregistrement tridimensionnel systématique du matériel a été privilégié. Les contraintes de délai ont néanmoins nécessité d'adapter le protocole dans l'objectif de traiter l'intégralité du gisement, en passant de l'enregistrement en trois dimensions à un enregistrement par $1 / 4 \mathrm{~m}^{2}$, partiel ou total selon les secteurs (zones hachurées, fig. 1.3). C'est ainsi que 12477 éléments d'industrie lithique, 11307 restes de faune, 236 pièces d'industrie osseuse, 35 éléments de parure et neuf restes humains ont été positionnés en 3D. Les densités obtenues sur le matériel prélevé selon cette méthode peuvent dépasser 1200 pièces $/ \mathrm{m}^{2}$. Le matériel qui n'a pas fait l'objet de cet enregistrement a été démonté par $1 / 4 \mathrm{~m}^{2}$. Il n'a pour l'heure pas été quantifié mais représente un volume de plusieurs milliers de pièces, toutes catégories confondues. Enfin, environ onze tonnes de sédiment ont été prélevées afin de procéder au tamisage intégral des couches archéologiques. Le tamisage en cours livre une quantité considérable d'esquilles osseuses, en majorité carbonisées, et lithiques, de fragments de lamelles à dos et d'éléments de parure. Ces ensembles seront quantifiés avec précision lors des phases d'étude.

\section{La faune}

La faune issue de la couche 14 présente un très bon état de conservation. Les états de surface sont plus dégradés dans les couches 4/22, 13 et 19. Elle est dominée par le cheval et le renne, complétée par du Boviné, des Carnivores (renard, loup), des Léporidés et probablement de l'oiseau. Certains restes de loup portent des traces de découpe impliquant, au moins, le prélèvement de la peau. La qualité de la représentation des éléments squelettiques et des surfaces osseuses offrent l'opportunité de reconstituer les modalités d'acquisition et de traitement du gibier exploité par ces magdaléniens. Les analyses de ces restes fauniques s'avèrent prometteuses à plusieurs titres. Elles viendront éclairer des pratiques cynégétiques d'un secteur encore peu exploré de ce versant de la Roche pour une période qui a été partiellement documentée par E. Turner (Combier et Montet-White, 2002) dans le secteur P16 des fouilles de J. Combier entre 1968 et 1974.

\section{L'industrie lithique}

Les tendances, issues des observations de fouille, donnent un premier aperçu de l'industrie lithique. L'outillage, abondant, utilise des supports laminaires réguliers et rectilignes. Il comprend burins (dièdres, le plus souvent), grattoirs, becs, perçoirs et micro-perçoirs, outils doubles, ainsi que des lames à retouches écailleuses, légèrement couvrantes. Les objectifs de la production semblent principalement lamellaires. Des blocs de petits volumes, éclat ou burins polyédriques, sont exploités pour la production de supports transformés en lamelles à dos rectangles, à double troncature transversale (fig. $2, \mathrm{n}^{\text {os }} 1$ à 6 ).

\section{L'industrie osseuse}

La série regroupe des témoins en bois de renne, en os et en ivoire. Si les états de conservation et les états de surface sont globalement bons, ils sont très inégaux, allant parfois d'un extrême à l'autre, selon les secteurs du site et les couches archéologiques. Les objets finis se distinguent par des bipointes en bois de cervidé (fig. 2, $\mathrm{n}^{\text {os }} 7$ et 8 , inconnues dans les proches contextes jurassiens), des pointes à biseau simple (fig. $2, \mathrm{n}^{\text {os }} 9$ et 10 ), et des pointes en ivoire similaires à celles de la grotte Grappin, Isère, et de l'abri Croze, Ain (Malgarini 2014; Malgarini et al., sous presse). La fouille a également livré des fragments d'aiguilles à chas et de bâtons percés (dont un entier et de forme phallique). Les déchets en bois de renne sont particulièrement nombreux et souvent issus de gros modules (bois de chute de mâles adultes). Les déchets en os sont également bien identifiables avec des matrices de baguettes sur métapodes de cheval. Les baguettes en bois de renne dominent la catégorie des supports.

\section{La parure et l'art mobilier}

La parure se compose de plusieurs dents animales perforées ou décorées (craches de cervidé fig. 2, $\mathrm{n}^{\circ} 11$, incisive de renne, canine de renard, ...), de deux os hyoïdes découpés et perforés, d'éléments en ivoire et de vingt coquillages perforés (Cyclope neritea, fig. $2, \mathrm{n}^{\circ} 12$ et Homalopoma sanguineum). Une grande partie de ces objets, ainsi que des éléments en os, portent des séries d'incisions parallèles. Le tamisage d'un tiers du volume total de sédiments prélevés permet notamment d'ajouter à cet ensemble vingt et un coquillages perforés, un fragment d'os hyoïde perforé, un fragment de crache de cervidé et une perle discoïde en calcite.

\section{Les restes humains}

Dans le tiers nord-est de la fouille, huit fragments crâniens d'adulte ont été identifiés, en position stratigraphique intermédiaire, à la base de la couche 22 et au contact de la couche 14 . Une analyse anthropologique combinée à une date absolue permettra d'associer ou non ces fragments à l'occupation magdalénienne. Par ailleurs, la couche 14 livre une molaire déciduale, sans lien avec les fragments crâniens.

\section{Le travail en cours...}

Actuellement, le traitement primaire du mobilier (lavage de la faune et tamisage) est en cours. Le traitement de 
l'industrie lithique est en attente. L'industrie osseuse fait l'objet d'une étude préliminaire menée dans le cadre du rapport final d'opération. En l'état, les moyens déployés dans le cadre de l'opération préventive n'autorisent pas l'étude complète du site. Le contenu du rapport a été défini en fonction de ces moyens. Il s'agira dans un premier temps de dresser un bilan quantitatif complet associé à une analyse qualitative partielle pour chaque champ d'étude. Les travaux ultérieurs seront en mesure de proposer une étude complète du gisement.
Remerciements : Cette première présentation est l'occasion de remercier chaleureusement les intervenants et collaborateurs qui ont permis la bonne conduite de l'opération, en particulier les membres de l'équipe «Ethnologie préhistorique » (UMR 7041 ArScAn) pour leur précieuse expertise et leur disponibilité tout au long de la fouille, ainsi que les autorités en charge du suivi $\mathrm{du}$ dossier. De très vifs remerciements à l'ensemble de l'équipe Inrap (diagnostic et fouille) qui s'est impliqué sans compter pour tenter de comprendre et « venir à bout » de cette véritable profusion de vestiges.

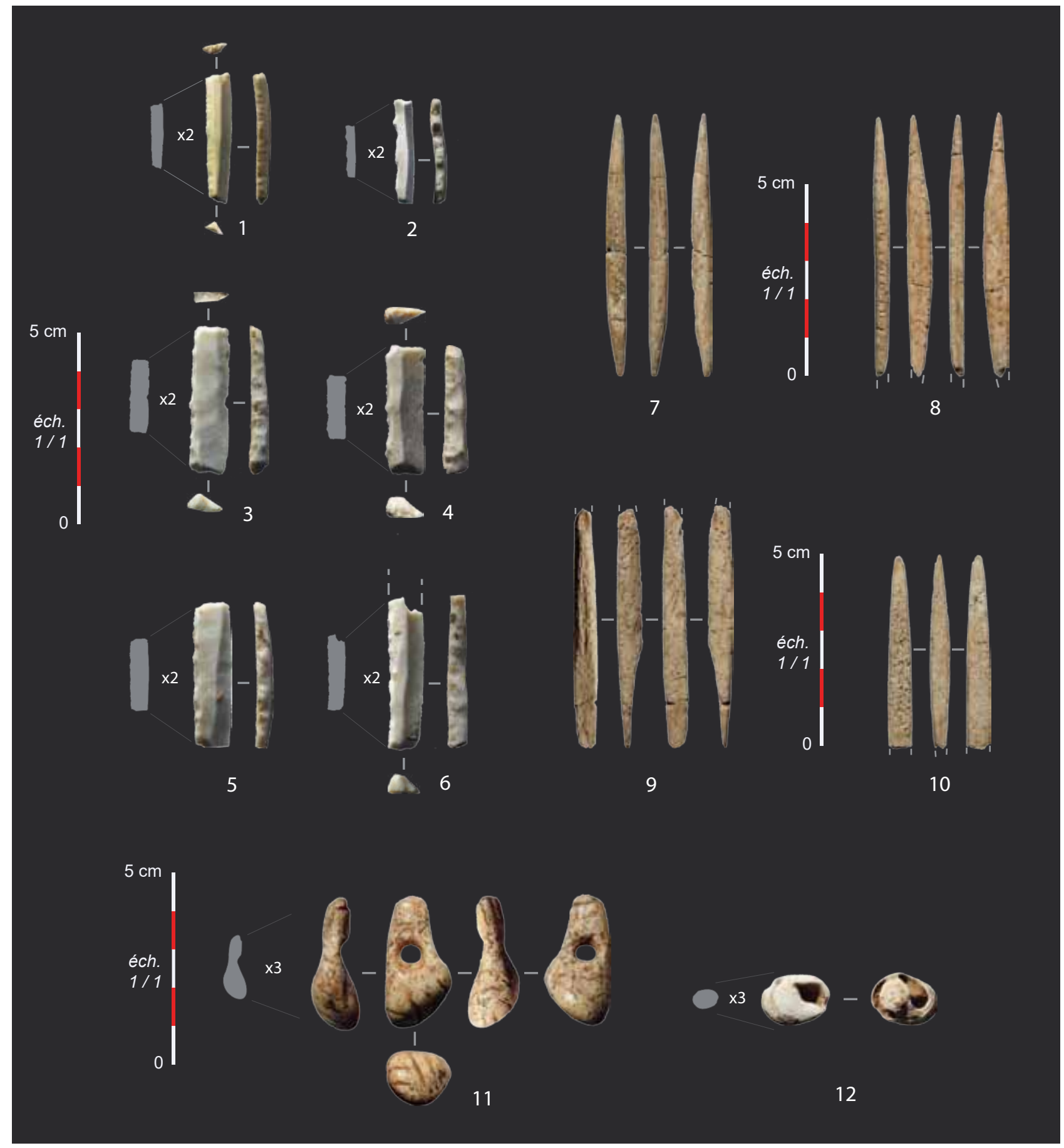

Fig. 2 - Industries lithiques, industries osseuses et parures. 1-6 : lamelles à dos (clichés J.-B. Lajoux); 7-8 : bipointes (clichés R. Malgarini); 9-10 : pointes à biseau simple (clichés R. Malgarini); $11:$ crache de cervidé; $12:$ Cyclope neritea (clichés R. Malgarini) 


\section{RÉFÉRENCES BIBLIOGRAPHIQUES}

Bayle G., Bemilli C., Connet N. (2011) - Apport de la fouille préventive en 2004 à la caractérisation des occupations aurignaciennes à Solutré (Saône-et-Loire, France), in F. Bon, S. Costamagno et N. Valdeyron (dir.), Haltes de chasse en Préhistoire. Quelles réalités archéologiques?, actes du colloque international (Toulouse,13-15 mai 2009), P@lethnologie, 3, p. 169 à 181.

Bemilli C., Bayle G. (2009) - Aurignacian Animal Exploitation at Solutré (Saône-et-Loire, France), in L. Fontana, F.-X. Chauvière et A. Bridault (éd.), In Search of Total Animal Exploitation: Case Studies from the Upper Palaeolithic and Mesolithic, actes du XVe Congrès de l'UISPP congress, session C61 (Lisbonne, 4-9 septembre 2006), Oxford, John and Erica Hedges, 2009 (BAR, International Series 2048), p. 37 à 47.

Combier J., Montet-White A., dir. (2002) - Solutré 1968- 1998, Paris, Société préhistorique française (Mémoire, 30), 281 p.

Connet N., Bayle G., Bemilli C., Kervazo B., Konik S., Julien M., Vanhaeren M. (2012) - Stratégies de subsistances des Aurignaciens de Solutré (Saône-et-Loire). Les apports de la fouille préventive de 2004, Gallia Préhistoire, 54 , p. 33-65.

Lajoux J.-B., Coll. Bemilli C., Malgarini R. (2015) - Bourgogne, Saône-et-Loire, Solutré-Pouilly, Route de la Roche. L'occupation magdalénienne à Solutré : nouvelles données acquises dans le cadre d'une évaluation archéologique, rapport de diagnostic, INRAP Grand Est sud, 83 p.

Malgarini R. (2014) - Les gisements magdaléniens dans le Jura et les Alpes $d u$ Nord et leurs industries osseuses, thèse de doctorat, université de Franche-Comté, Besançon, 2 vol., 484 et $182 \mathrm{p}$.

Malgarini R., Mevel L., Bereiziat G., Bodu P., Cupillard C., Debout G. Carquigny N. (sous presse) - Les faciès du Magdalénien moyen dans l'Est de la France : confron- tation et discussion des industries osseuses et lithiques, in C. Bourdier, L. Chehmana, R. Malgarini, M. PoltowicsBobak (dir.), L'essor du Magdalénien : aspects culturels, techniques et symboliques des faciès à navettes et à Lussac-Angles, actes de la séance de la Société préhistorique française (Besançon, 17 -19 octobre 2013).

Jean-Baptiste LAJOux

INRAP Grand-Est Sud, Centre archéologique de Besançon, 9, rue Lavoisier, F-25000 Besançon jean-baptiste.lajoux@inrap.fr

Romain MALgARINI UMR 7041 ArScAn, Ethnologie préhistorique, Maison de l'archéologie et de l'ethnologie, 21, allée de l'université, F-92023 Nanterre cedex romain.malgarini@gmail.com

Céline Bemilli UMR 7209 Archéozoologie, archéobotanique, INRAP Grand Ouest, centre archéologique de Grand Quevilly, 30, boulevard de Verdun, Immeuble Jean Mermoz F-76120 Grand-Quevilly celine.bemilli@inrap.fr

Grégory BAYLE UMR 7324 CITERES , INRAP Centre-Île-de-France, 32 , rue Délizy, F-93694 Pantin cedex gregory.bayle@inrap.fr 